\title{
Nonequilibrium charge transport through Falicov-Kimball structures connected to metallic leads
}

\author{
Martin Žonda and Michael Thoss \\ Institute of Physics, Albert-Ludwig University of Freiburg, \\ Hermann-Herder-Strasse 3, 79104 Freiburg, Germany
}

\begin{abstract}
Employing a combination of a sign-problem-free Monte Carlo approach and nonequilibrium Green's-function techniques, we study nonequilibrium charge transport in a model heterostructure, where a two-dimensional spinless Falicov-Kimball system is coupled to two noninteracting leads. We show that the transport characteristic depends sensitively on the electrostatic potential in the system and exhibits different properties for different phases of the Falicov-Kimball model. In particular, pronounced step-like changes of the current and transmission are observed at the phase boundaries, evident even on a logarithmic scale. Analyzing finite size effects, we find that with the method used a relatively small system can be utilized to address specific thermodynamic limits. Phenomenon of the localization is discussed as well.
\end{abstract}

\section{INTRODUCTION}

The study of strongly correlated electron systems (SCESs) has been a field of intensive research for several decades. In recent years, the investigation of outof-equilibrium phenomena has received particular attention. Experimental studies in this context comprise a variety of different techniques and architectures including time-dependent problems in quantum dots ${ }^{1-\underline{8}}$, molecular bridges and nano-wires ${ }^{9}-14$, layered systems, junctions, and hetero-structures $15-18$, as well as dynamics of quenched cold atoms in optical latices $\frac{19}{22}$ or ultrafast electronics $23-25$ and pump-probe experiments on the charge density wave materials $(\mathrm{CDW})^{26-28}$. Theoretical investigations have focused on open questions related, e.g., to the formation of nonthermal steady state ${ }^{29,30}$, dynamical phase transitions, and hidden metastable phases revealed by driving $31-35$ as well as to the evolution of open quantum systems in general ${ }^{36,37}$.

To study these open questions, a variety of theoretical methods have been employed. Some of them are rather versatile, e.g., methods based on nonequilibrium Green's functions ${ }^{38-41}$; however, they often rely on significant approximations, which limit their validity to certain parameter regimes, such as, e.g., weak coupling $42-44$. Other methods, e.g., nonequilibrium dynamical mean-field theory (DMFT) ${ }^{45}, 46$, require so-called nonequilibrium impurity solvers $4-54$. Although there is rapid progress in the development of these solvers, their application is still numerically very demanding. Especially challenging is the study of long-time evolution including nonequilibrium steady-state properties. It is, therefore, important to study special cases of SCES models, which can be addressed by less demanding, but still exact methods. The results of such studies not only help us to gain a deeper understanding of nonequilibrium phenomena, but can also serve as testing tools for addressing more challenging systems. An example of such a model, which in recent years played an important role in the studies of nonequilibrium SCES, is the Falicov-Kimball model (FKM).
The spinless version of the FKM was initially introduced as a limiting case of the Hubbard model, where one electron species is localized ${ }^{55}$. Later, but independently and with spin degrees of freedom, the model was used for the metal-insulator transitions in rare-earth and transition-metal compounds 56 . The FKM has been used successfully for the description of numerous equilibrium phenomena (for overviews see Refs. $57-60$ ), for different properties of layered systems $61-64$ and for problems related to transport, time evolution and nonequilibrium steady states $30,31,65-71$. From the conceptual point of view, the most profound advantage of the FKM is the fact that it is exactly solvable in the limit of infinite dimension (infinite coordination number) by means of DMFT ${ }^{72}$ and that for finite lattices it can be addressed by an exact, sign-problem-free Monte Carlo (MC) method ${ }^{73}-76$. In addition, both these methods can be extended to nonequilibrium without the necessity to introduce any approximation $45,46,71,77$.

In the present paper, we introduce a combination of the sign-problem-free $\mathrm{MC}$ method with a nonequilibrium Green's-function technique, which allows us to address the nonequilibrium steady state of a composite structure consisting of a finite FKM system (or its various generalizations) and infinite leads. An important advantage of this method is that it can be applied to any lattice geometry and particle filling in finite dimension. The only limiting factor is the total size of the system. The method is therefore especially useful for lattices with only few atomic layers in one or two directions (layered systems) connected to semi-infinite leads 61.78 . For such geometries, the method can describe large enough systems to approach the thermodynamic limit.

Inspired by the recent rapid progress in understanding the equilibrium and nonequilibrium properties of the two-dimensional (2D) FKM $66,71,79,80$ we primarily concentrate on charge transport through finite square FKM lattices driven by voltage differences of the lead potentials (for illustration see Fig. 11). Our focus is on how different phases of the FKM influence the transport characteristics and how the nonequilibrium steady state influences 


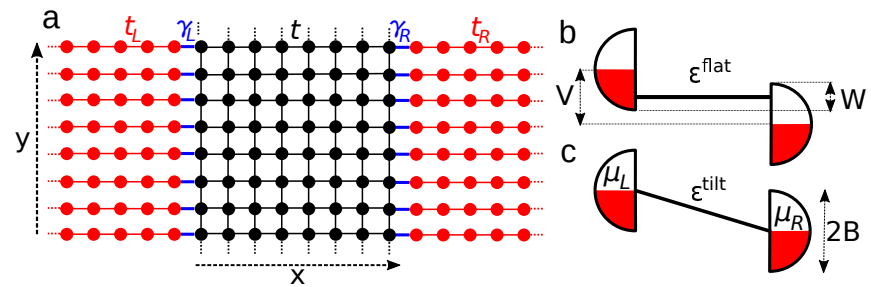

Figure 1. (a) Schematic picture of the heterostructure. The black part represents the FKM system with nearest neighbor hopping $t$. The red parts are noninteracting leads with hopping $t_{L, R}$ and the hybridization interaction with hopping $\gamma_{L, R}$ is indicated in blue. (b-c) Illustration of the introduced voltage bias with two limiting cases of the potentials taken into account in the present paper. The elliptic DOSs of the leads are shifted by $\epsilon_{L, R}$ and the condition $\mu_{L, R}=\epsilon_{L, R}$ is used to keep the bands half filled at any applied voltage. The system potential used is either (b) flat $\varepsilon^{\text {flat }}=-U / 2$ or (c) tilted $\varepsilon^{\text {tilt }}$ (see the text).

the typical phases of the FKM. Although, in the present paper, we primarily concentrate on square lattices, we show that the results are also valid for more general layered systems.

The rest of the paper is organized as follows. In Sec. II we introduce the model and outline the methodology. In Sec. III] we discuss two different choices of the electrostatic potential in the system. We analyze the equilibrium properties of the FKM necessary for understanding the nonequilibrium steady state for the two choices in Sec. IIIA 1 and Sec. IIIB 1. In Secs. IIIA 2, IIIB 2 the respective transport properties of the FKM are analyzed. We show that the potential has a crucial effect on the transport properties and that the current is very different in the ordered and disordered phase. The effects of the system size are discussed in Secs. III A 3. III B3. Sec. IV] concludes with a summary.

\section{MODEL AND METHOD}

We consider a composite structure consisting of a large but finite central system described by the spinless FKM and two infinite leads. The Hamiltonian of this heterostructure reads $H=H_{S}+\sum_{l=L, R} H_{\text {lead }}^{l}+H_{h y b}^{l}$ (for illustration see Fig. 1(a)), where

$$
\begin{aligned}
H_{S}= & -\sum_{i, j} t_{i j} d_{i}^{\dagger} d_{j}+U \sum_{i} f_{i}^{\dagger} f_{i} d_{i}^{\dagger} d_{i} \\
& +\sum_{i} \varepsilon_{i}^{d} d_{i}^{\dagger} d_{i}+\sum_{i} \varepsilon_{i}^{f} f_{i}^{\dagger} f_{i},
\end{aligned}
$$

represents the central system.

Here, the first term describes spinless electrons moving on a lattice. The geometry of the lattice and the hopping amplitudes are defined by the hopping matrix t. In the present paper, we focus on square lattices with a constant nearest-neighbor hopping amplitude $t$. We assume periodic boundary conditions at the edges perpendicular to the system-lead interface. This mitigates the finite-size effects in the $y$ direction. Most of the results presented below have been obtained for lattice size $L=L_{x} \times L_{y}=20 \times 20$. Nevertheless, the method can be used for any Hermitian matrix $\mathbf{t}$. The second term represents a Coulomb-like local interaction between the localized $f$ particle and itinerant $d$ electron on the same lattice site. The last two terms describe the position-dependent potentials that act on both particle species. This potential can be, in principle, influenced by the leads and, depending on the interpretation of the FKM, can differ for $f$ and $d$ particles 81 . However, throughout this paper we will assume that the $f$ particles are also spinless electrons and are, therefore, affected by the potential equally as $d$ electrons. Correspondingly, we set $\varepsilon_{i}^{f}=\varepsilon_{i}^{d}$. This choice is advantageous with respect to the interpretation of the FKM as a limiting case of the Hubbard mode $1^{55}$. Moreover, we fix the number of $f$ particles to $N_{f}=L / 2$. This reflects the fact, that only $d$ electrons are directly coupled to the leads, which act as infinite reservoirs, and that the model does not contain a hybridization between the two particle species. The specific profiles of the potential are discussed in Sec. III.

The leads are taken to be noninteracting

$$
H_{\text {lead }}^{l}=-t_{l} \sum_{\langle m, n\rangle}\left(c_{l, m}^{\dagger} c_{l, n}+c_{l, n} c_{l, m}^{\dagger}\right)+\epsilon_{l} \sum_{n} c_{l, n}^{\dagger} c_{l, n},
$$

where $\langle m, n\rangle$ is a sum over the nearest-neighbor sites, $t_{l}$ is the hopping for lead $l=L, R$ and $\epsilon_{l}$ represents an energy shift. Finally, the hybridization between lead $l$ and the system is described by the term

$$
H_{\text {hyb }}^{l}=-\gamma_{l}\left(\sum_{\langle i, n\rangle} c_{l, n}^{\dagger} d_{i}+d_{i}^{\dagger} c_{l, n}\right),
$$

with $\gamma_{l}$ being the corresponding hopping parameter. We assume that the hybridization is turned on adiabatically starting in the distant past with a decoupled state, where both leads and system had been in thermal equilibrium. Note, that the initial conditions play an important role because the FKM is an integrable model with the occupations of the $f$ particles being integrals of motion ${ }^{82}$. The importance of initial conditions was already demonstrated in nonequilibrium DMFT studies of the time evolution of the FKM after a quench ${ }^{30}$. We partially address this problem by using two different system potentials.

It is advantageous to rewrite the full model in a different basis using the fact that the $f$-particle occupation numbers $f_{i}^{\dagger} f_{i}$ commute with the entire Hamiltonian and are thus good quantum numbers. This allows us to replace $f_{i}^{\dagger} f_{i}$ by its eigenvalues $w_{i}=0$ or $w_{i}=1$ and write a partial Hamiltonian for a particular classical configuration $w$. This significantly simplifies the problem. The 
system Hamiltonian for a chosen configuration $w$ reads

$$
H_{S}^{w}=\sum_{i, j} h_{i j} d_{i}^{\dagger} d_{j}+\sum_{i} \varepsilon_{i}^{f} w_{i}=\sum_{\alpha} \tilde{\varepsilon}_{\alpha} \tilde{d}_{\alpha}^{\dagger} \tilde{d}_{\alpha}+\sum_{i} \varepsilon_{i}^{f} w_{i},
$$

where $h_{i j}=\left(U w_{i}+\varepsilon_{i}\right) \delta_{i j}-t_{i j}$ and where we have diagonalized the first term by formally applying a simple unitary transformation

$$
d_{i}=\sum_{\alpha} \mathcal{U}_{i \alpha} \tilde{d}_{\alpha}, d_{j}^{\dagger}=\sum_{\beta} \tilde{d}_{\beta}^{\dagger} \mathcal{U}_{\beta i}^{\dagger},
$$

with $\tilde{\varepsilon}_{\alpha}$ being the eigenvalues of matrix $\mathbf{h}$ and where matrix $\mathcal{U}$ consists of the related eigenvectors arranged in columns. The matrix $\mathcal{U}$ can be computed numerically for a finite system and it can be chosen to be real. We can formally apply an equivalent transformation also to lead terms

$$
c_{l, n}=\sum_{k} \mathcal{V}_{l, n k} \tilde{c}_{l, k}, c_{l, n}^{\dagger}=\sum_{k} \tilde{c}_{l, k}^{\dagger} \mathcal{V}_{l, k n}^{\dagger}
$$

leading to

$$
\begin{aligned}
& H_{\text {lead }}^{l}=\sum_{k} \tilde{\epsilon}_{l, k} \tilde{c}_{l, k}^{\dagger} \tilde{c}_{l, k}, \\
& H_{\text {hyb }}^{l, w}=\sum_{\alpha, k} \tilde{\gamma}_{l, k \alpha} \tilde{c}_{l, k}^{\dagger} \tilde{d}_{\alpha}+\tilde{\gamma}_{l, \alpha k}^{\dagger} \tilde{d}_{\alpha}^{\dagger} \tilde{c}_{l, k},
\end{aligned}
$$

where $\tilde{\gamma}_{l, k \alpha}=-\gamma_{l} \sum_{\langle i, n\rangle} \mathcal{V}_{l, k n}^{\dagger} \mathcal{U}_{i \alpha}$ keeps track of the actual geometry of the system-lead interface and therefore can not be taken constant.

The transformed Hamiltonian (44)-(6) describes a relatively simple noninteracting model where both the "level" energies $\tilde{\varepsilon}_{\alpha}$ and the hybridization strength $\tilde{\gamma}_{l, \alpha k}$ are functions of the configuration $w$. The nonequilibrium transport in this kind of models is a well studied problem ${ }^{83}$ and the exact form of the steady-state Green's functions is given by ${ }^{38}$

$$
\begin{aligned}
\mathbf{G}^{r, a}(E) & =\mathbf{g}^{r, a}(E)+\mathbf{g}^{r, a}(E) \boldsymbol{\Sigma}^{r, a}(E) \mathbf{G}^{r, a}(E), \\
\mathbf{G}^{<}(E) & =\mathbf{G}^{r}(E) \boldsymbol{\Sigma}^{<}(E) \mathbf{G}^{a}(E) .
\end{aligned}
$$

Here, $\mathbf{G}^{r(a)}$ is the retarded (advanced) Green's function of the whole structure, $\mathbf{G}^{<}$is the lesser Green function and $\mathbf{g}^{r(a)}(E)$ is the retarded (advance) Green's function of the bare system with components

$$
g_{\alpha \beta}^{r, a}(E)=\frac{\delta_{\alpha \beta}}{E-\tilde{\varepsilon}_{\alpha} \pm i 0} .
$$

The total tunneling self-energies $\boldsymbol{\Sigma}^{r, a,<}=\boldsymbol{\Sigma}_{L}^{r, a,<}+\boldsymbol{\Sigma}_{R}^{r, a,<}$ have the components

$$
\Sigma_{l, \alpha \beta}^{r, a,<}(E)=\sum_{k} \tilde{\gamma}_{l, \alpha k}^{*} \mathbf{g}_{l, k}^{r, a,<}(E) \tilde{\gamma}_{l, k \beta},
$$

where $\mathbf{g}_{l}^{r, a,<}(E)$ is the noninteracting Green's function of the isolated lead $l$. To simplify the analysis, we assume leads with zero hopping in the direction parallel to the system-lead interface, i.e., the leads consist of identical semi-infinite chains with hopping $t_{l}$. The exact self-energies for this choice of leads can be found analytically ${ }^{84,85}$ and read

$$
\begin{aligned}
\Sigma_{l, \alpha \beta}^{r, a}(E) & =\Lambda_{l, \alpha \beta}(E) \mp \frac{i}{2} \Gamma_{l, \alpha \beta}(E), \\
\Sigma_{l, \alpha \beta}^{<}(E) & =i \Gamma_{l, \alpha \beta}(E) f_{l}\left(E-\mu_{l}\right), \\
\Gamma_{l, \alpha \beta}(E) & =2 \pi \gamma_{l}^{2} \mathcal{U}_{\alpha \beta}^{\left\{s^{l}\right\}} \rho_{l}(E), \\
\Lambda_{l, \alpha \beta}(E) & =\frac{2 \gamma_{l}^{2}}{B^{2}} \mathcal{U}_{\alpha \beta}^{\left\{s^{l}\right\}}\left(E-\mu_{l}\right), \\
\mathcal{U}_{\alpha \beta}^{\left\{s^{l}\right\}} & =\sum_{i \in\left\{s^{l}\right\}} \mathcal{U}_{\beta i}^{\dagger} \mathcal{U}_{i \alpha},
\end{aligned}
$$

where $\left\{s^{L, R}\right\}$ are the sets of system lattice positions at the left and right interfaces, $f_{l}(E)$ is the Fermi function, and $\mu_{l=L, R}$ is the chemical potential of the leads. The leads are characterized by a smooth surface density of states (DOS):

$$
\rho_{l}(E)=\frac{2}{\pi B^{2}} \sqrt{B^{2}-\left(E-\epsilon_{l}\right)^{2}},
$$

with band half width $B=2 t_{l}$ centered around $\epsilon_{l}$.

The tunneling current for fixed configuration $w$ has a compact Landauer-Büttiker form, 38

$$
I^{w}=\int \frac{d E}{2 \pi} \mathscr{T}^{w}(E)\left[f\left(E-\mu_{L}\right)-f\left(E-\mu_{R}\right)\right],
$$

with the transmission function

$$
\mathscr{T}^{w}(E)=\operatorname{Tr}_{\mathrm{d}}\left[\boldsymbol{\Gamma}_{L}(E) \mathbf{G}^{r}(E) \boldsymbol{\Gamma}_{R}(E) \mathbf{G}^{a}(E)\right],
$$

where the trace goes over the $d$-electron subsystem. In the following we set $\mu_{L}=\epsilon_{L}$ and $\mu_{R}=\epsilon_{R}$, which corresponds to half-filled lead bands and we introduce a finite voltage drop as $V=\mu_{L}-\mu_{R}$ with condition $\mu_{L}=-\mu_{R}$.

It is worth noting that the numerical solution of Eq. (7), which represents a system of linear equations for complex matrices of the size $L \times L$, together with the matrix multiplication in Eq. (12), present the bottleneck for the numerical evaluation. This is because, despite the broadening provided by the self-energy, the Green's functions can still contain sharp $\delta$-function-like features, especially at low temperatures and for strong interaction $U$. The evaluation of the current therefore requires a dense mesh of energies $E$ (see e.g. Fig. 6(h)). Fortunately, this part can be easily parallelized.

So far, we have addressed only the problem for a fixed configuration $w$. The calculation of average values of any operator $\hat{O}$ for the $d$ electrons requires a trace over the $f$ particles as well. Considering the above stated initial condition and assuming that the leads are not affected by the system, the trace over the classical degrees of freedom reduces to a simple formula

$$
\langle\hat{O}\rangle=\frac{1}{Z} \sum_{w} e^{-\beta F(w)}\langle\hat{O}\rangle_{d}
$$


where

$$
F(w)=-\frac{1}{\beta} \sum_{\alpha} \ln \left[1+e^{-\beta \tilde{\varepsilon}_{\alpha}}\right]+\sum_{i} \varepsilon_{i}^{f} w_{i},
$$

with $Z=\sum_{w} e^{-\beta F(w)}$ being the partition function and $\langle.\rangle_{d}$ being the trace over the $d$-electron subsystem for fixed $w^{73}$. E.g., the total (bulk) DOS of the isolated system is defined as $\operatorname{DOS}(E)=\operatorname{Tr}_{w} \sum_{\alpha} \delta\left(E-\varepsilon_{\alpha}\right) / L$ and the DOS of the coupled heterostructure is defined as $\operatorname{DOS}(E)=\operatorname{Tr}_{w} \operatorname{Tr}_{d} i\left[\mathbf{G}^{r}(E)-\mathbf{G}^{a}(E)\right] / 2 \pi L$, where $\operatorname{Tr}_{w} \equiv \frac{1}{Z} \sum_{w} e^{-\beta F(w)}$. Calculation of the averages of operators which reflect the real-space distributions requires us to retrace the unitary transformation. For example, the average $d$-electron occupation on a particular site $i$ of a coupled system is given by

$$
\left\langle n_{d}^{i}\right\rangle=-i \operatorname{Tr}_{w} \int d E \sum_{\alpha, \beta} \mathcal{U}_{i \alpha} \mathcal{U}_{\beta i}^{\dagger} G_{\alpha \beta}^{<}(E)
$$

and the local density of states (LDOS) can be calculated using

$$
\begin{aligned}
\operatorname{LDOS}_{i}(\mathrm{E})= & \frac{i}{2 \pi L} \operatorname{Tr}_{w}\left[\sum_{\alpha, \beta} \mathcal{U}_{i \alpha} \mathcal{U}_{\beta i}^{\dagger} G_{\alpha \beta}^{r}(E)\right. \\
& \left.-\sum_{\alpha, \beta} \mathcal{U}_{\alpha i}^{\dagger} \mathcal{U}_{i \beta} G_{\alpha \beta}^{a}(E)\right] .
\end{aligned}
$$

The power of this approach lies in the fact that the averaging in Eq. (13) can be performed by a simple signproblem-free Monte Carlo method ${ }^{73}-\underline{76}$. In this method, the classical configuration $w$ is updated following the Metropolis algorithm, where the difference in the free energy, Eq. (14), for different $w$ is used to build a Markov chain. To obtain the free energy a single-particle quantum problem is solved exactly in every Monte Carlo step by numerical diagonalization which can be done efficiently ${ }^{86}$. It is worth noting that this method is not limited to the equilibrium or steady state as the time evolution is accessible as well ${ }^{71}$.

Because of the broadening of the Green's functions, provided by the coupling to the leads, we can also calculate the zero-temperature characteristics. In this case, the Monte Carlo averaging can be avoided. All that is needed, is the correct $f$-electron ground-state configuration. We calculate the ground states using a simple simulated annealing method 87 , which is similar to the Monte Carlo method presented here, with the difference that the ground-state energy $E_{G S}(w)=\sum_{\alpha=1}^{N_{d}} \tilde{\varepsilon}_{\alpha}+\sum_{i} \varepsilon_{i}^{f} w_{i}$ for a particular configuration is used instead of $F(w)$ to calculate the weights in the annealing process.

\section{RESULTS}

Because of the vastness of the parameter space we have restricted the present paper to symmetric couplings of $\gamma=\gamma_{L}=\gamma_{R}=2 t$ and $4 t$. These values provide a sufficient broadening of the transmission function even for zero temperature $(T=0)$. We also use relatively broad DOSs for the leads with the half width $B=B_{L}=B_{R}=10 t$ or $20 t$, which are, however, still narrow enough to study the effects related to a finite band.

As already stated, the profiles of $\varepsilon^{d}$ and $\varepsilon^{f}$ can be influenced by the leads and should be, in general, calculated self-consistently. Following Ref. 88 we instead consider two limiting cases: a flat potential, where $\varepsilon_{i}^{\text {flat }}=$ $\varepsilon_{i}^{d}=\varepsilon_{i}^{f}=-U / 2$ (Fig. 1(b)) for every lattice point $i$; and a tilted potential $\varepsilon_{i}^{\text {tilt }}=\varepsilon_{i}^{d}=\varepsilon_{i}^{f}=-U / 2+n_{x}\left(\epsilon_{L}-\right.$ $\left.\epsilon_{R}\right) /\left(L_{x}+1\right)$ (Fig. 1(c)), where $n_{x}$ numbers the layers from left to right. Note, that for the purpose of this paper we use the term "layer" for a single chain parallel to the interface ( $y$ direction). Both potential profiles are centered around $-U / 2$. This choice fixes the half-filling condition at zero chemical potential for the whole system but not in every particular layer. We assume that the realistic profile should be in between these two cases. These two choices can be also understood as two different initial conditions. This follows from the fact that the $f$ electrons are integrals of motion. Their distribution is fixed by the initial thermalization. The flat potential describes a situation where the system was initially completely isolated from the leads and for the tilted potential the voltage drop heavily influenced the system potential before the coupling was switched on.

\section{A. Flat potential}

We start our analyses with the flat electrostatic potential, which we suppose is more realistic than the tilted one, at least for a large system. Our primary goal is to address two rather general questions, namely, how the coupling to the leads and the finite voltage influence the phases of the FKM and how these phases, if still present, influence the charge transport in the heterostructure. Therefore we first discuss the phase diagram of the spinless FKM.

\section{Phase diagram and equilibrium properties}

The general phase diagram of the spinless FKM is profoundly rich and includes stable exotic orderings such as stripes and various charge segregations $60,75,89,90$. Moreover, the phase diagram is fairly complicated even at half filling, where the FKM with flat potential is at low temperature always in the CDW phase for any bipartite lattice ${ }^{74,91}-94$. In Fig. 2(a), a simplified version of the phase diagram is plotted for an isolated 2D FKM system. The three main regions that are crucial for our study of transport are the low-temperature ordered phase (OP), the disordered phase for weak interaction $(\mathrm{DPw})$, and the disordered phase for strong interaction (DPs). The 

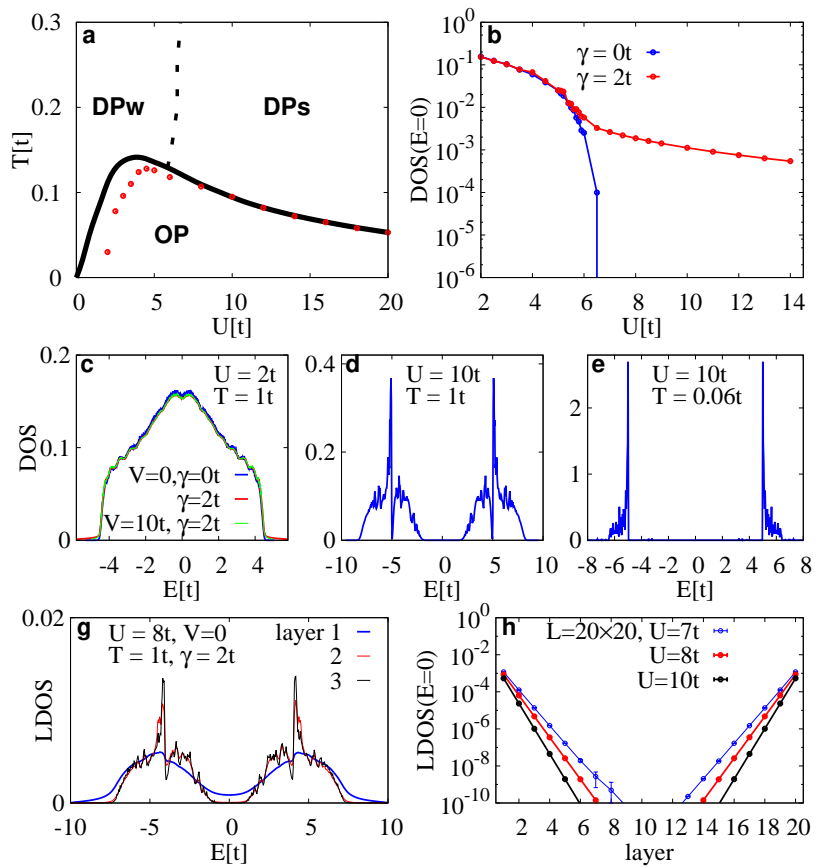

Figure 2. (a) Simplified phase diagram of the isolated system with ordered phase (OP) and disordered phases in weak $(\mathrm{DPw})$ and strong (DPs) interaction regimes. The lines represent phase boundaries for the flat potential $\varepsilon^{\text {flat }}$. The dots show an estimate for $\varepsilon^{\text {tilt }}$ at $V=5 t$, taken from the position of the maxima of the approximated specific heat ${ }^{73}$ for $L=20 \times 20$. (b) Equilibrium $d$-electron DOS at $E=0$ for coupled (red) and decoupled (blue) systems calculated in the vicinity of $\mathrm{DPw}$-DPs transition for the flat system potential for $T=1 t$ and plotted as a function of $U$. (c-e) Illustration of the typical $d$-electron DOS for DPw (c), DPs (d), and OP (e) from the phase diagram (a). Blue lines have been calculated for the isolated system, and red and green lines in (c) have been calculated for the coupled system. (g) Local DOS of the coupled system at equilibrium for the first three system layers parallel to the interface. (h) Local DOS of the coupled system calculated at the Fermi level for all system layers and various values of $U$ in the Mott regime. In all cases the system size is $L=20 \times 20$.

equilibrium $d$-electron DOS in OP (Fig. 2(e)) contains a CDW gap with a width equal to $U$ at zero temperature. The width of the CDW gap does not change with increasing temperature, but rather the gap is filled in by subgap states $\underline{95}-\underline{-97}$. The gap is completely closed in DPw (Fig. 2(c)) and a narrower Mott gap stays open between Hubbard-like bands in DPs (Fig. 2(d)). Nevertheless, a CDW pseudo gap can be present in the DOS even at high temperatures.

Because of the absence of the gap in the DOS (Fig. 2(c)), the DPw was initially considered to be metallic in two dimensions ${ }^{73,74}$, but recent studies on bigger clusters showed that in the thermodynamic limit it is actually an Anderson insulator phase and that for any finite system the $\mathrm{DPw}$ also contains a crossover from the Anderson insulator to a broad weak localization phase at weak interactions ${ }^{79,98}$.

It is worth noting that the phase diagram is in principle even more complicated. For example, for some parameters it is possible to have a CDW gap in the DOS but a nonzero DOS at the Fermi leve $1^{95,97,99}$. Here we focus on the phases mentioned above.

We first investigate the influence of the leads in equilibrium. The phase boundary between $\mathrm{OP}$ and disordered phases is not affected by finite $\gamma$. This is because the phase transition is mostly driven by the ordering of $f$ particles and, as their occupations are integrals of motion, the full system always keeps the thermalized $f$-particle distribution of the decoupled system. This is, however, not necessarily true for the DPs-DPw phase boundary, which mostly reflects the transition from finite to zero gap in the DOS of $d$-electrons.

We show a comparison of the total DOS at the Fermi level $(E=0)$ for the isolated and coupled system (with $\gamma=2 t$ ) in Fig. 2(b). It is evident, that the coupling closes the Mott-like gap otherwise clearly developed in the DPs. The reason is the leaking of the leads metallic density of states into the system. This is illustrated in Fig. 2(g), where we plot the LDOS calculated for the first three system layers parallel to the interfaces. The LDOS of the first layer (blue line) is strongly broadened by the coupling. The effect quickly vanishes with increasing distance from the interface. The decay is exponential for strong interaction, which is in compliance with DMFT studies 58,61. We show this in Fig. 2(g), where we plot the dependence of $\operatorname{LDOS}(E=0)$ on the distance from the left interface for $L=20 \times 20$ and three values of $U$. The leaking of the leads' DOS into the system is the dominant contribution to the $\operatorname{LDOS}(E=0)$ for strong interaction (Fig. 2(g)), because there the Mott-like gap is clearly developed for the isolated system even for finite lattice sizes. Therefore, the exponential decay of the $\operatorname{LDOS}(E=0)$ with the distance from the interfaces reflects directly the vanishing influence of the leads. Note, that the decay clearly depends on $U$ and it is therefore more complicated for intermediate and weak coupling where, in addition, the gap is not yet opened even for a decoupled system. It is therefore challenging to analyze quantitatively the effect of the leads on DPs-DPw transition. Nevertheless, we will readdress the question in the next section, where we discuss the transport properties.

The broadening of the DOS of the coupled system allows us to define the averaged generalized inverse participation ratio $(\mathrm{gIPR}) 100,101$

$$
\operatorname{gIPR}(E)=\operatorname{Tr}_{w} \frac{\sum_{i} \operatorname{LDOS}_{i}^{2}(E, w)}{\operatorname{DOS}^{2}(E, w)},
$$

for the coupled system without the necessity to regularize the possible $\delta$-functions. The inverse participation ratio and its generalization are used for the identification of localization in isolated strongly correlated electron systems in equilibrium ${ }^{100}-102$. Here we show that it can be useful also for the analysis of an open system. 

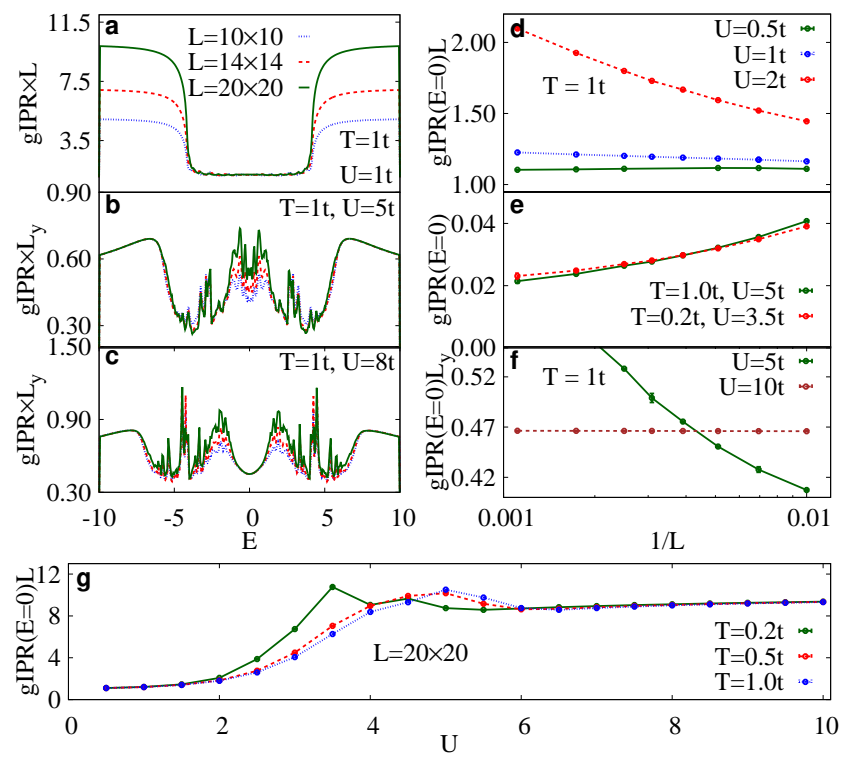

Figure 3. (a-c) The generalized inverse participation ratio calculated for the coupled system and for different $U$ and $L$. The results plotted in (a) are multiplied by $L$ and in (b,c) are multiplied by $L_{y}$. (d-f) The size scaling of the generalized inverse participation ratio at the Fermi level in three regimes discussed in the main text. (g) The generalized inverse participation as a function of $U$ for different temperatures and $L=20 \times 20$. All figures show equilibrium state $(V=0)$ with coupling $\gamma=2 t$ and half bandwidth of the bands set to $B=10 t$.

The gIPR scales as $1 / L$ for completely itinerant system states. Considering the exponential decay of the influence of the lead DOS shown in Fig. $2(\mathrm{~g})$, the ratio scales as $\sim 1 / L_{y}$ for energies within the gap as here the dominant contribution comes from the interfaces. The ratio should converge to a finite value with increasing $L$ for localized system states and should be constant for a sufficiently large system and strong localization. The inverse participation ratio is therefore ideal for studying the complicated transition from the Fermi gas at $U=0$ to the Mott-like phase for $U \gtrsim 6 t^{79}$.

Fig. 3 shows an analysis of the gIPR for the coupled system in equilibrium. Subplots (a-c) depict its energy dependence for weak $(U=1 t)$ intermediate $(U=5 t)$ and strong interaction $(U=8 t)$ and different lattice sizes. For weak interaction the gIPR scales as $\sim 1 / L$ (note that the data in Fig. 3(a) are multiplied by $L$ ) for a broad range of energies around zero and significantly deviates from this scaling only for energies outside the range of the isolated-system DOS where the main contributions come from the leads' broadening. This is shown also in Fig. 3(d), where we plot the scaling of the gIPR at the Fermi level for small $U$. The value of $\operatorname{gIPR}(E=0) \times L$ is practically constant for $U \lesssim t$ and one can conclude that in this region the states are predominantly delocalized for a finite number of layers.

For strong interaction $U$ (Fig. 3 (c,f)), gIPR scales as
$1 / L_{y}$ in the region of the Mott gap, as well as for energies outside the full width of the DOS of the isolated system, as expected for a gapped system. The scaling in the intermediate interaction region $(3 t \lesssim U \lesssim 6 t)$ is not that straightforward. The dependence of $\operatorname{gIPR}(E=0)$ on $U$ shows a maximum in this region evident in Fig. $3(\mathrm{~g})$ and the scaling (Fig. 3(e)) seems to point to a finite value of $\operatorname{gIPR}(E=0)$ in the thermodynamic limit. Both these results indicate an Anderson-like localization for states near $E=0$ which is in compliance with a previous study of the isolated system by Antipov et al.,$\underline{79}$. However, a more thorough analysis on a significantly larger system is necessary to confirm this conjecture.

Note, that the change of the temperature does not play a significant role if $T$ is much higher than the critical temperature $T_{c}(U)$ of the order-disorder transition (Fig. 3(g)). However, this changes when the temperature approaches $T_{c}$. The sensitivity of $\operatorname{gIPR}(E=0)$ on temperature is most notable in the intermediate interaction regime. Nevertheless, we can conclude, that all main phases of the FKM are still present even for the coupled heterostructure which includes different localization regimes in the $\mathrm{DPw}$.

\section{Transport properties}

We start our discussion of the transport properties for the flat potential by considering a finite $L=20 \times 20$ system and first explain its most important features. Only afterwards we discuss the effects of the system size on the transport in detail (Sec. IIIB 3).

The real-space distribution of the $d$ electrons of the decoupled system either is homogeneous or forms a CDW pattern. In both cases the flat potential fixes the average $d$-electron occupancy to $L_{y} / 2$ for every layer. The nonequilibrium distribution of the coupled system shown in Fig. 4 is somewhat different. Following the respective chemical potential of the coupled leads, the distribution is elevated or lowered close to the interface for $T=0$. In the central region, the distribution approaches $L_{y} / 2$. At high temperatures, the normalized occupations for very high voltage have nearly a linear slope (see the open black circles in Fig. (4). A significant spatial difference at high voltage can be seen also in the LDOS shown in Fig. 4(c,d). The shifted leads' surface densities of states $\rho_{L}(E)$ and $\rho_{R}(E)$ broaden the system LDOS asymmetrically. As expected, the effect is most notable at the interfaces. For example, the part of the system LDOS located outside the width of $\rho_{l}(E)$ of the neighboring lead is broadened only by the temperature.

Fig. [5 shows examples of $I-V$ characteristics of the FKM system for three values of $U$ which, according to Fig. 2(a), represent the weak-interaction $(U=2 t)$, intermediate-interaction $(U=5 t)$ and strong-interaction $(U=10 t)$ case. The results in Fig. 5 (a-c) have been calculated using the coupling $\gamma=2 t$ and a bandwidth of $B=10 t$. The current in Fig. 5(d) was obtained for the 

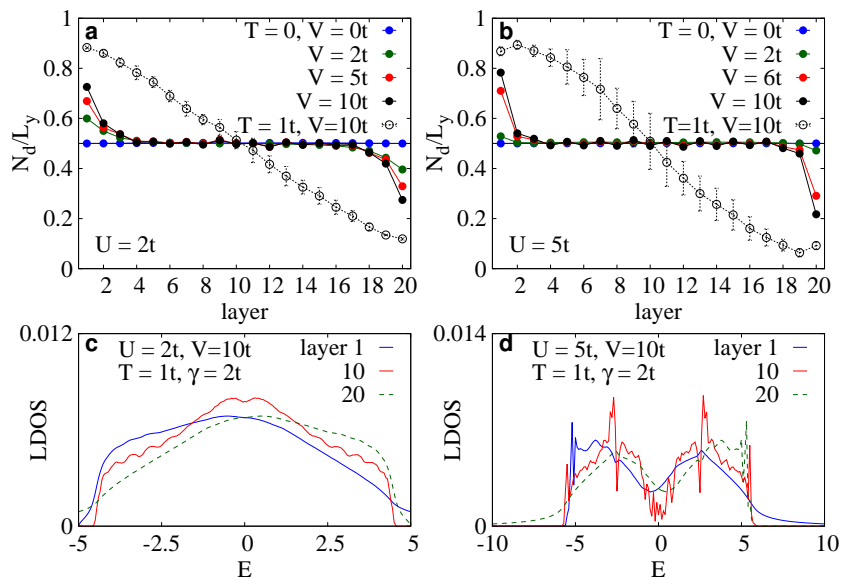

Figure 4. (a,b) Normalized $d$-particle occupations per layer parallel to the system-lead interfaces for a coupled system with $\gamma=2 t, B=10 t$, flat system potential, with interactions (a) $U=2 t$, and (b) $U=5 t$. The filled circles show the zero temperature results for various $V$. The open circles represent the high-temperature result for $T=1 t$ and $V=10 t$. (c,d) The nonequilibrium LDOS calculated at the interfaces and central part of the system for high temperature $(T=1 t)$ and high voltage $V=10 t$.

same ratio $\gamma / B$ but a broader band, $B=20 t$. The two high-temperature cases $T=1 t$ and $T=0.2 t$ illustrate how the decreasing temperature influences the transport in the disordered phases. Temperature $T=0.1 t$ addresses the transport just below the phase boundary and $T=0 t$ shows the system without thermal excitations. To understand the $I-V$ characteristics, we also present in Fig. 6 the related transmission functions for selected voltages. The transmission functions are particularly useful as they contain the most detailed information about elastic transport. The nonequilibrium transmission for small $V$ resembles the equilibrium DOS. Similarly to the total DOS, and despite the strong modulation of the LDOS (Fig. 4(c,d)), the transmission function changes significantly with voltage only when $V \gtrsim B$ (see, e.g., case $V=15 t$ in Fig. G $(\mathrm{a}, \mathrm{d}, \mathrm{g}))$. However, the most important features considering transport are much more pronounced in the transmission function than in the DOS or LDOS already in equilibrium. This is particularly true for the pseudo-gap around $E=0$, which is evident even for high temperature and weak interaction (compare Fig. 4(c) with Fig. 6(a)). A similar statement is true also for the typical sub-gap structures for intermediate and strong interaction (Fig. 6(b,c)). This suggests that the $f$-electron configurations responsible for the closing of the CDW gap at high temperatures have low transmission which can be again attributed to a significant localization of the $d$ electrons.

Most of the features of the current can be understood by following the two windows confining the integration over the relevant part of the transmission function in Eq. (11). The first one is the Fermi function window,
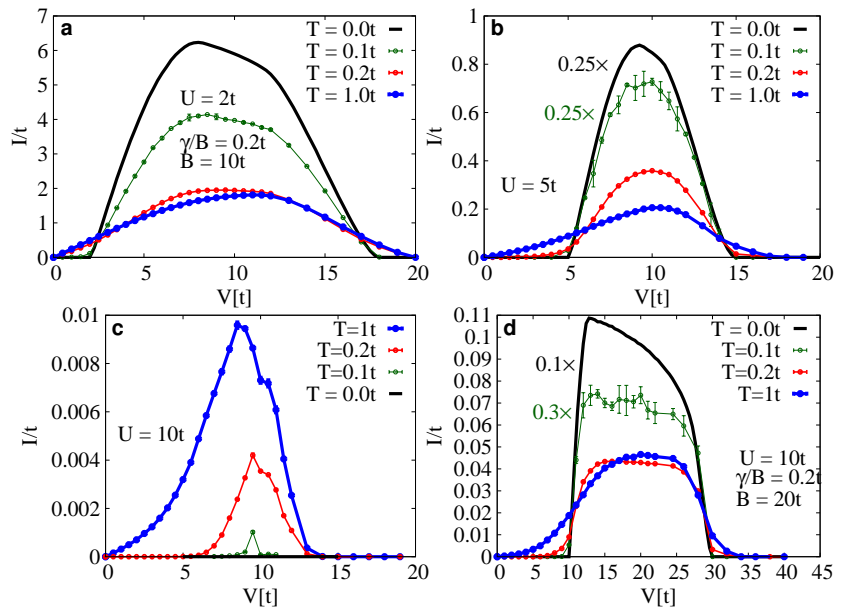

Figure 5. Current-voltage characteristics for flat system potential, system size $L=20 \times 20$, coupling $\gamma / B=0.2$ and parameters (a) $U=2 t, B=10 t$; (b) $U=5 t, B=10 t$; (c) $U=10 t, B=10 t$; and (d) $U=10 t, B=20 t$. Note that the curves for the two lowest temperatures in (b) are scaled by factors 0.25 and in $(\mathrm{d})$ by factors 0.1 and 0.3 to improve the visibility.

the width of which is proportional (and at zero temperature identical) to $V$. The second one is the band window $W=2 B-V$ (see Fig. $1(\mathrm{~b})$ ), which restricts the width of the transmission function (Eq. (10)).

The basic profile of the current for $T=1 t$ is quite similar for all values of $U$. The current increases up to $V \sim B$ and then decreases to zero. The increase of the current reflects the broadening of the Fermi function window. Around $V=B$ the two windows change their roles because for $V>B$ it holds that $W<V$ and the decrease of the current reflects the decrease of $W$. This is a typical effect of the finite bandwidth of the leads, but their shape and the width of the DOS of the isolated system play a role as well.

As expected, the maximal current decreases with increasing $U$ because the $d$ electrons become more localized. However, whereas the current maximum is increasing with decreasing temperature for $U=2 t$ and $5 t$, it seems to rapidly vanish for $U=10 t$ in Fig. [5(c). This is again a direct consequence of the finite bandwidth of the leads. For $U=10 t, V>10 t$, and $B=10 t$ and regardless of the broad Fermi window, the transmission function fenced by $W$ covers only the sub-CDW gap structures in Fig. 6(c,f), which vanish with decreasing $T$. Therefore, the current drops rapidly with decreasing $T$ and also for $V>B$. To analyze the influence of the Hubbardlike satellites on the current in the strong-interaction regime, we have calculated the current also for broader bands $B=20 t$ while keeping the ratio $\gamma / B=0.2$ (see Fig. [5(d)). The results confirm the trend of increasing the current maximum with decreasing temperature.

An opposite trend can be observed in the region where $V<U$ (and $V \sim 2 B$ ). For $U=2 t, T=1 t$ and small 

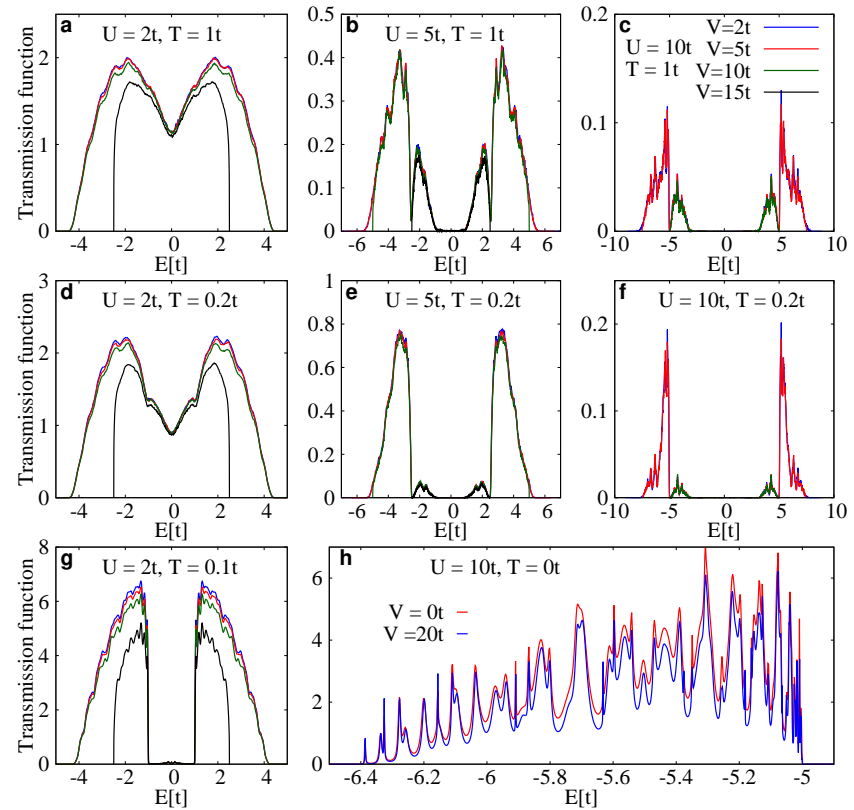

Figure 6. (a-g) Transmission function for the flat system potential for coupling $\gamma=2 t$, band half-width $B=10 t$ and different values of $U, T$, and $V$. (h) Detail of the Hubbardlike satellite for $\gamma=4 t$, band half width $B=20 t$ and strong interaction $U=10 t$ plotted for the $V=0 t$ and $20 t$ cases.

voltages, the current depends approximately linearly on $V$ but decreases with both increasing $U$ and decreasing $T$. The decrease is a consequence of the opening and deepening of the CDW pseudo gap with decreasing temperature and opening of the Mott pseudo gap with increasing $U$ in the transmission function. This can be seen in Fig. 6 (g) where the transmission function is almost negligible in the interval $|E|<U / 2$ already for $T=0.1 t$ and even at weak interaction. The increase of the Fermi window to $V \sim U$ leads to a sharp increase of the current in Fig. 5 as now the window covers the states outside the CDW gap as well.

We illustrate the different trends in different voltage regions again in Fig. 7 (a,b), where we show the current as a function of the temperature for voltages $V \lesssim U$ and $V \sim B$ (close to optimal voltage with maximal current). The opposite trends are clearly demonstrated for temperatures near and below the DP-OP transition. For $V \lesssim U$, the current drops around the critical temperature. This change is steplike for weak interaction (where the high-temperature phase is $\mathrm{DPw}$ ) and gradual on the logarithmic scale otherwise. On the other hand, the current increases dramatically just below the critical temperature for $V \sim B$. Interestingly, this means that the current can signal the order-disorder phase transition of the system differently for low and optimal voltage.

This result can be understood by focusing on three tendencies. First, the Fermi window becomes sharper with decreasing temperature, which puts more "weight" on the transport through the central regions of the DOS. The
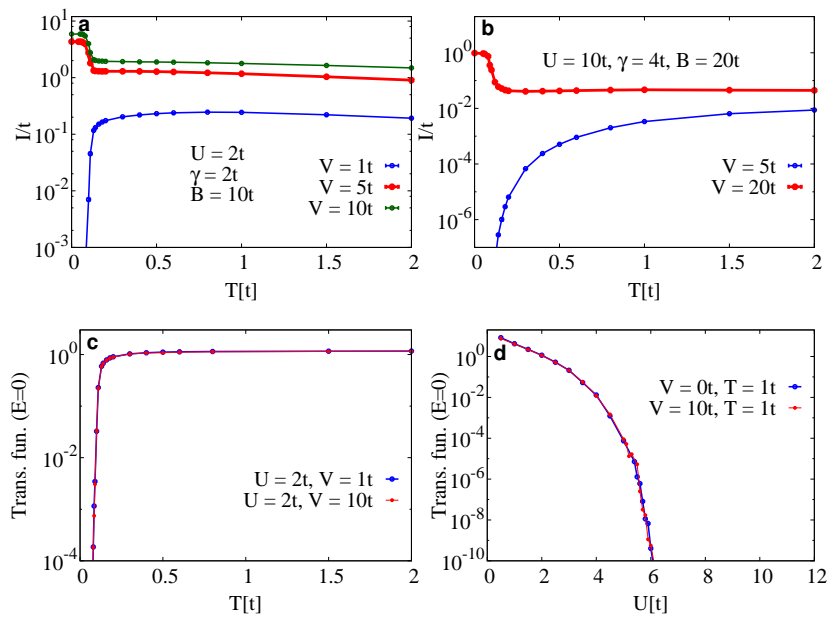

Figure 7. (a-b)Temperature dependence of the current for voltages below and above the respective width of the CDW gap. (c) Transmission function $E=0$ as a function of temperature for $U=2 t$ and $V=1 t, 10 t$. (d) Transmission function at $E=0$ as a function of $U$ for $T=1 t$ and $V=0,10 t$.

second tendency is that the Hubbard-like maxima in the transmission function are becoming narrower and higher with decreasing temperature (compare Fig. 6(c) and (h)). The sharp Fermi window, therefore, ensures a sharp increase of the current in the interval of voltage that covers the Hubbard-like satellites. This is most profoundly seen in Fig. 5(d). For zero temperature, the current sharply increases in the range $U<V \lesssim 13 t$, which covers exactly the Hubbard-like satellites in the transmission function (see the detail in Fig. 6(h)). For higher voltages, the current decreases. This is initially caused by a small decrease of the transmission function shown in Fig. 6(h) but mostly by closing of the band window $W$. The third tendency is the rapid disappearing of the CDW in-gap states with decreasing temperature. For small voltages $(V<U)$ and low temperatures, this leads to a drop in the current. The difference in the temperature dependencies between the weak and strong intersection reflects the fact that for strong interaction there is already a Mott gap in the DOS even above the critical temperature.

We can therefore conclude that the three typical phases of the 2D FKM have a qualitatively different influence on the charge transport properties reflected in current characteristics. Moreover, the transition between the phases can be seen directly from the transmission function. We illustrate this in Fig. 7( $(\mathrm{c}, \mathrm{d})$ where the transmission function at $E=0$ is plotted as a function of temperature (c) and $U$ (d). Note, that at equilibrium the transmission function is qualitatively equivalent to the conductance and it is relatively insensitive to voltage at $E=0$ (see the overlap of the curves in Fig. 7( $(\mathrm{c}, \mathrm{d})$ ). Therefore, the sudden drop of the transmission over several magnitudes that signals the critical temperature in Fig. 77(c) points to a qualitative change in the character of transport between $\mathrm{DPw}$ and OP. Even more interesting is the 

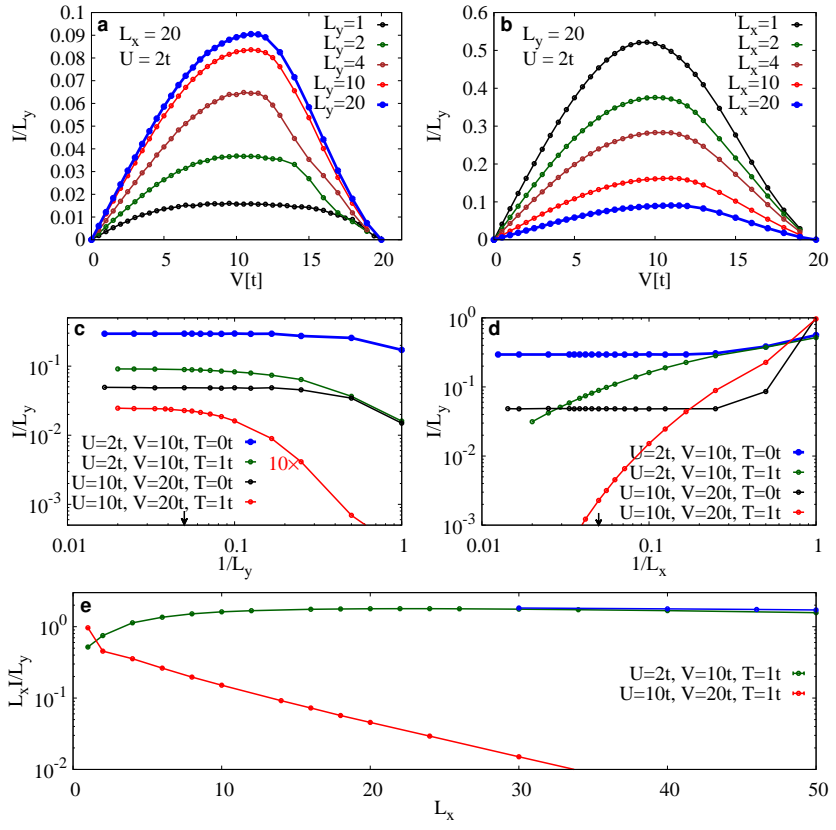

Figure 8. Current densities plotted: (a) as a function of voltage for a system with fixed $L_{x}=20$ and different $L_{y}$ and $T=1 t$; (b) as a function of voltage for a system with fixed $L_{y}=20$ calculated for different numbers of layers $L_{x}$ and $T=1 t$; (c) as a function of inverse linear size $1 / L_{y}$ for $L_{x}=20, \gamma=0.2 B, U=2 t, U=10 t$, optimal $V$, and zero temperature, where the current density for $U=10 t, T=1 t$ was multiplied by 10 for the sake of clarity; (d) as a function of inverse linear size $1 / L_{x}$ for a system with fixed $L_{y}=20$, $\gamma=0.2 B, U=2 t, U=10 t$ and optimal $V$, for high and zero temperature, and (e) for a system with $L_{y}=20$ (green and red) and $L_{y}=30$ (blue), where the current densities are multiplied by $L_{x}$.

exponential drop of the transmission with increasing $U$ in Fig. 7(d). Although the total nonequilibrium DOS plotted in Fig. 2(b) shows that the Mott gap in DPs is closed by coupling to the leads, the drop of the transmission function points to a similar critical $U$ as the DOS of the decoupled system even after introducing a relatively high voltage $(V=10 t)$. This reflects the rapid decrease of the influence of the leads on the LDOS (Fig. 2(g,h)) with the distance from the interface. Consequently, the DPw-DPs phase boundary seems to be unaffected by the coupling to the leads and clearly manifests itself in the transport properties.

\section{System size effects}

In this section, we consider the effect of the system size on the transport properties. In Fig. 8(a), the current densities $\left(I / L_{y}\right)$ are plotted as functions of the voltage for high temperature $(T=1 t)$, flat potential, weak coupling $U=2 t$, and different $L_{y}$, starting with a single chain $\left(L_{y}=1\right)$. The current density rapidly saturates with increasing $L_{y}$. This is a general feature as we show in Fig. 8(c), where the current densities are plotted for weak $(U=2 t)$ and strong interaction $(U=10 t)$ at zero and high temperature as functions of $1 / L_{y}$. The chosen voltages are close to their optimal values, where the biggest differences in current density for various $L_{y}$ are observed. All four curves are practically saturated at $L_{y}=20$. This means that the results obtained for $L_{y}=20$ are actually a good representation of a system in the limit $L_{y} \rightarrow \infty$.

We next focus on the other dimension, $L_{x}$. Fig. $8(\mathrm{~d})$ shows examples of the current densities for fixed $L_{y}=20$ but different $L_{x}$ starting with a single layer. For zero temperature, the current density rapidly saturates with increasing $L_{x}$. This is because for the flat potential, the localized $f$ particles at zero temperature form a periodic (checkerboard) potential and there are no further sources of scattering. We conclude that the system $L=20 \times 20$ is sufficient for addressing transport through an infinite zero-temperature 2D FKM system at half filling.

The situation is different for $T=1 t$ where the current density decreases with the increasing $L_{x}$ (Fig. 8(d)). Nevertheless, the size dependence is different for weak and strong interaction $U$. In (Fig. $8(\mathrm{e})$ ), we plot the same current densities multiplied by $L_{x}$ and as a function of $L_{x}$. Whereas for strong interaction the current density drops much faster than linearly, for $U=2 t$ the scaled current density initially increases and then seems to saturate with $L_{x}$. Note, that there is actually a small decrease of $I L_{x} / L_{y}$ for $L_{x}>30$; however, this is related to fixed value of $L_{y}$. We confirmed the saturation by studying the dependence for $L_{y}=30$. Interestingly, this linear dependence of the current density on the system size together with almost linear $I-V$ characteristics for $V \lesssim 5 t$ in $\mathrm{DPw}$ points to a metal-like behavior. This might come as a surprise as it was recently shown that 2D FKM is an insulator in all its phases ${ }^{79}$, but it is actually consistent with the analysis of the gIPR for both isolated ${ }^{79}$ and coupled system (Fig. 3) which show a strong delocalization of the density of states for finite systems at weak interactions.

We analyze this for the case of high voltage by studying again the gIPR. Although the mutual shift of the $\rho_{L}$ and $\rho_{R}$ also introduces a spatial asymmetry in the LDOS, qualitative tendencies are still obvious. Figs. 9(a,b) show the gIPR profile for $E$ relevant for the transport at $V=10 t$ for weak interaction and $V=20 t$ at strong interaction. Figs. 9(c-f) show its finite-size scaling for chosen energies. The wide plateau in the energy profile of the weak coupling which scales roughly with $1 / L$ points to a prevailing delocalization. However, the scaling is not as convincing as for the equilibrium case. This may point to a stronger localization or could be a consequence of the discussed shift of the LDOS. The scaling is much more clear for the strong interaction $(U=8 t)$. Figs. 9(b,d) show that gIPR scales with $L_{y}$ only within the Mott gap and outside the normal width of the DOS of the isolated system, where the broadening of the edge layers dominates. The gIPR is practically constant for 

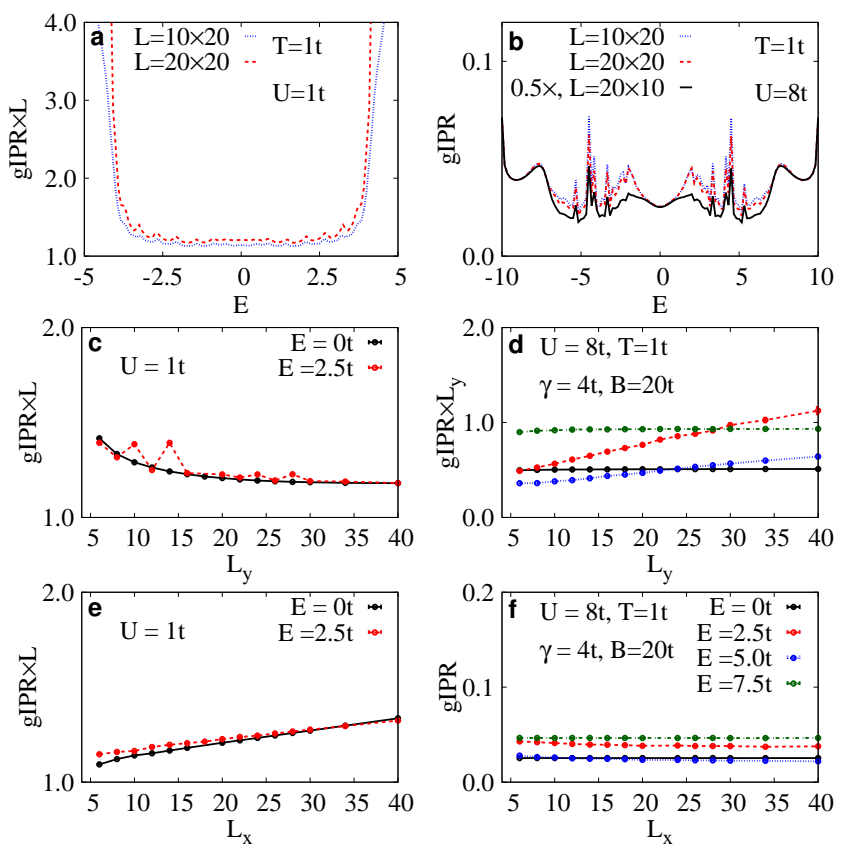

Figure 9. (a,b) The generalized inverse participation ratio for weak $(U=1 t)$ and strong interaction $(U=8 t)$ and large voltage drop. (c,d) The linear size scaling of the gIPR on $L_{y}$ for fixed $L_{x}=20$ and the densities at various energies. (e,f) The linear size scaling of the gIPR on $L_{x}$ for fixed $L_{y}=20$. The weak-interaction limit is plotted for $V=10 t, \gamma=2 t$, and $B=10 t$. The strong-interaction case is shown for $V=20 t$, $\gamma=4 t$, and $B=20 t$.

all energies plotted in Fig. 9)(f) including the energies within the isolated system DOS range. This points to a strong localization of all states relevant for the transport which explains the exponential drop of the current with the system width in Fig. 8(e).

\section{B. Tilted potential}

\section{Phase diagrams}

The phase diagrams of the tilted potential are in general more complex than for the flat one. Because the tilt is introduced before the coupling is switched on, the $f$-particle distribution is thermalized already under the influence of the electrostatic potential $\varepsilon^{\text {tilt }}$. The interplay of tree different energy scales, namely, the hopping term $-t$, potential $\varepsilon_{i}^{\mathrm{tilt}}$, and $U$, leads to a rich ground state summarized in the simplified phase diagram in Fig. 10. (a). The ground-state $f$-particle configurations are a mixture of segregated ${ }^{90,103}$ and checkerboard phase (CheP)[see Fig. 10(b-d)]. Note that the phase boundary between pure CheP and mixed phase (MixP) is stable. We have observed only small changes (below 5\%) with increasing system lattice size. On the other hand, the boundary between pure segregated phase (SegP) and MixP is relevant
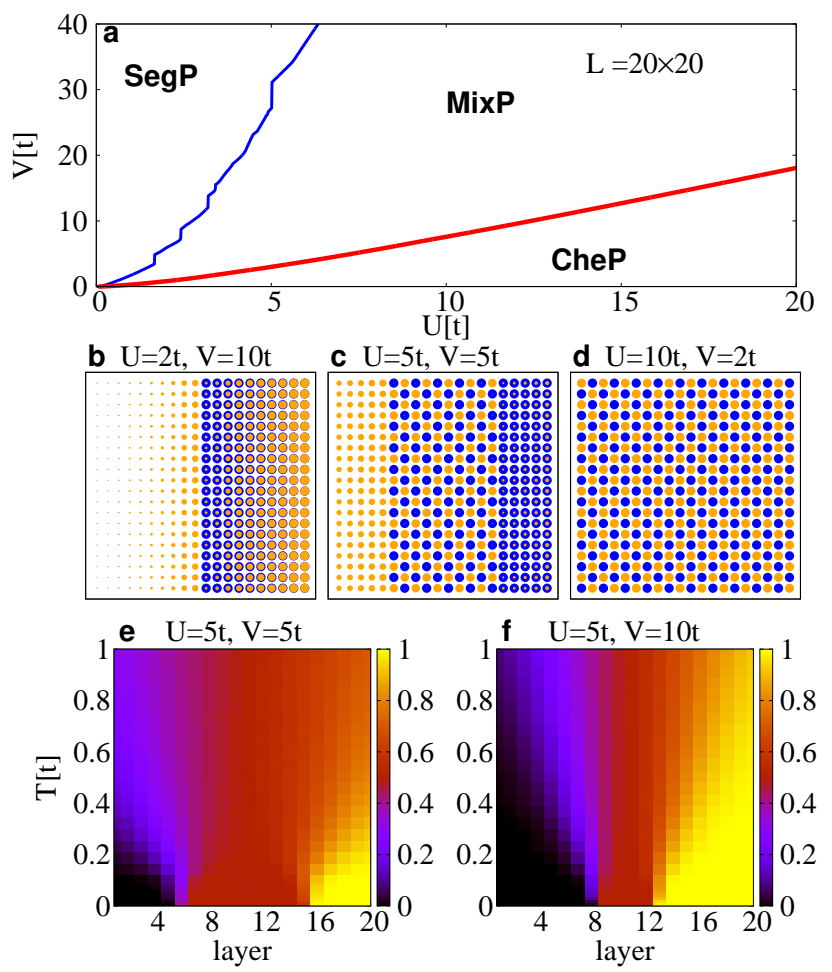

Figure 10. (a) Ground-state phase diagram for tilted potential and $L=20 \times 20$. Note, that the boundary between CheP and MixP is stable, but the boundary between MixP and SegP depends on the system size. (b-d) The representative ground-state configurations of $f$ particles (blue filled circles) and $d$ electrons (orange filled circles) for the decoupled system representing $\operatorname{SegP}(b), \operatorname{MixP}(c)$ and $\mathrm{CheP}(d)$. (e,f) Examples of the normalized $f$-particle occupations $\left(N_{f} / L_{y}\right)$ per layer for tilted potential, lattice $L=20 \times 20$ and parameters $\gamma=0, U=5 \mathrm{t}$, and $V=5 t$, and $V=10 t$ respactively.

only for $L=20 \times 20$. This boundary shifts to higher $V$ with increasing lattice size and for $L_{x} \rightarrow \infty$ and finite voltages the pure SegP might not exist at all.

It was shown before that the spinless FKM at neutral filling $\left(N_{f}=N_{d}\right)$ and in the vicinity of the half-filling condition contains different ordered phases for the same parameters but at different temperatures 75,104 . The potential tilt leads to a similar, although less pronounced situation. In Fig. 2(a) we show the estimated critical temperatures for the OP-DP transition (red filled circles) calculated for $V=5 t$ and $L=20 \times 20$. The segregation starts to form well above this transition. This can be seen in Fig. 10(e,f), where examples of the normalized $f$-particle occupation per layer parallel to the interfaces are plotted for a range of temperatures. The estimated critical temperatures in Fig. 2(a) are therefore related to the formation of the checkerboard pattern in the central region of the lattice. Consequently, the biggest difference between the phase boundaries for $\varepsilon^{\text {flat }}$ and $\varepsilon^{\text {tilt }}$ plotted in Fig. 2(a) is in the parameter regime where $V \gtrsim U$. In this regime, the checkerboard region is much smaller 

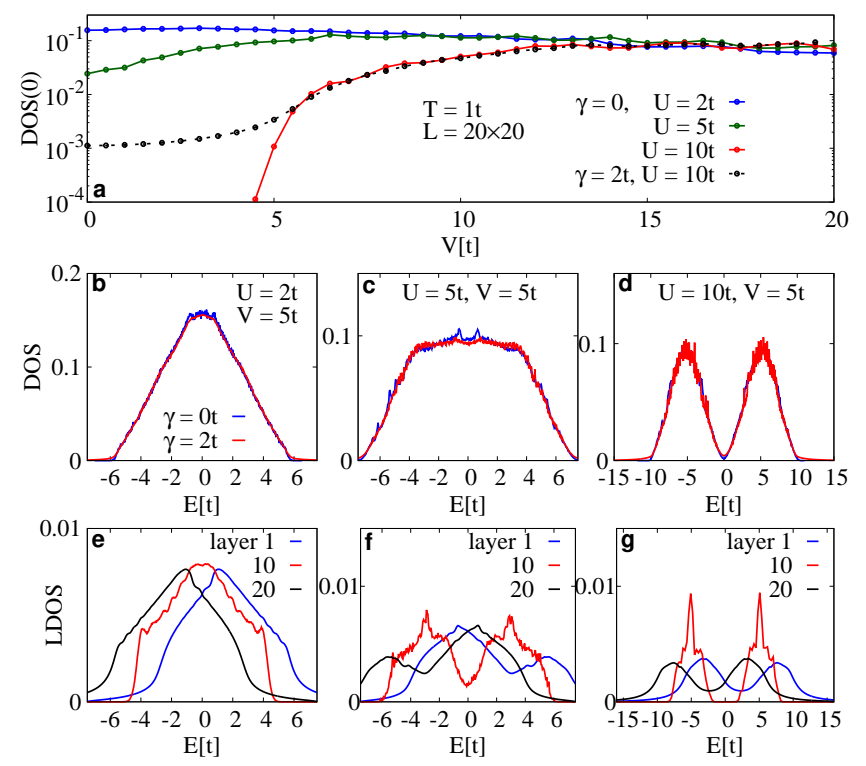

Figure 11. (a) DOS of $d$-electrons at $E=0$ for a system with tilted potential calculated as a function of voltage drop at $T=$ $1 t$ for a decoupled $(U=2 t, 5 t, 10 t)$ and coupled scenario $(U=$ $10 t, \gamma=2)$. (b-g) Examples of DOS for tilted potential and different model parameters calculated for decoupled (blue) and coupled (red) system with $L=20 \times 20$. (e-g) Local densities of states calculated for layers at the edges and in the center of the coupled system. The parameters are the same as in (b-d).

than the segregated one. Moreover, because for fixed parameters the total size of the checkerboard region increases with increasing $L_{x}$ the phase boundary in this region is not saturated yet for $L=20 \times 20$. We have observed that with increasing lattice size the boundary approaches the one for $\varepsilon^{\text {flat }}$. Nevertheless, for a finite system and tilted potential, the finite voltage can influence the OP-DP transition even before the coupling is turned on.

Because of the segregation, the tilt has a dramatic effect on the system even at high temperatures. This can be seen in the dependence of the DOS on $U$ for $E=0$ plotted in Fig. 11(a). A sufficiently tilted potential can close the well-developed Mott gap even for a decoupled system and strong interaction ( $U=10 t$ in Fig. 11(a)). The finite coupling closes the gap in the entire range of $V$ but the increase of the DOS at the Fermi level as a consequence of increasing tilt is still visible. We have observed that introducing the tilt has often a similar effect on the DOS as has the reduction of $U$ for the flat potential. It closes the pseudo gap, and the typical subCDW gap structures, usually present for strong interactions even above critical temperature, are merging with the Hubbard-like satellites (compare Fig. 11(b-d) with Fig. 2(c,d)).

The role of the segregation in this effect even at high temperatures can be understood by following the exam-
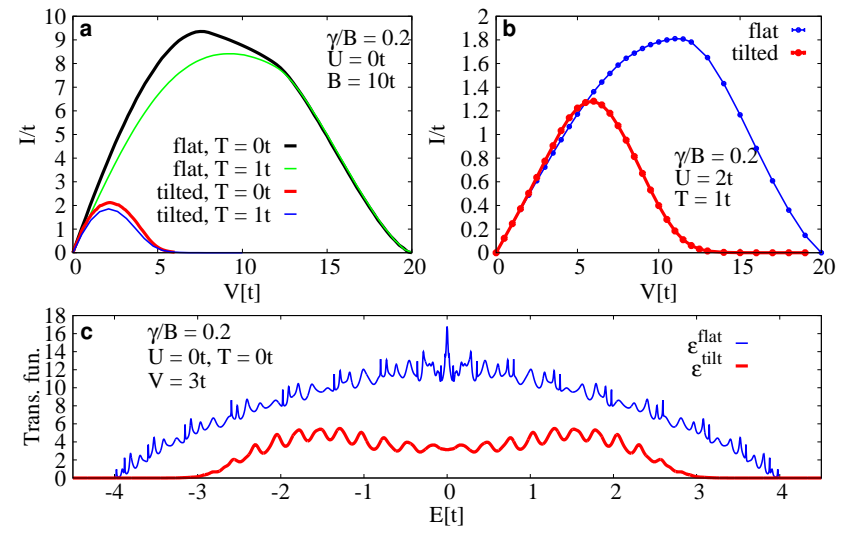

Figure 12. (a-b) Comparisons of current - voltage characteristics calculated for flat and tilted potential at high temperature $T=1 t$ and $0 t$. The left figure shows the noninteracting case, and the right one was calculated for $U=2 t$. The other parameters are $\gamma=2 t, B=10 t$, and $L=20 \times 20$. (c) Comparison of the transmission functions for the noninteracting system with flat and tilted potential for small voltage $V=3 t$.

ples of the LDOS for particular system layers in Fig.11(eg). Fig. 11(e) shows the LDOS of the coupled system for $U=2 t$ and $V=5 t$, where the ground state is completely segregated. All LDOSs resemble the DOS of a weakly interacting system, where the absence or presence of $f$ particles at the interfaces manifests itself by a constant shift competing with $\varepsilon_{i}^{\text {tilt }}$. For $U=2 t$ and $V=5 t$ (Fig. 11)(f)), the LDOS in the center region has a profile typical for the disordered phase at intermediate coupling, but the effect of the segregation is still obvious at the interfaces. As there is no segregation for $U=10 t$ and $V=5 t$ the "center" of $\rho_{L}(E)$ and $\rho_{L}(E)$, respectively, and the system LDOS at interfaces are almost aligned $\left(\epsilon_{L} \cong \varepsilon_{1}^{\text {tilt }}+U / 2\right.$ and $\varepsilon_{L_{x}}^{\text {tilt }}+U / 2 \cong \epsilon_{R}$ ), therefore LDOSs are shifted mostly by $\varepsilon^{\text {tilt }}$ and otherwise resemble a broadened DOS of the strongly interacting disordered phase.

\section{Transport properties}

As already shown above, the tilt of the potential can have a profound effect on the electron distribution. A similar result was obtained for the Hubbard model by Okamoto $\stackrel{88}{ }$. However, in that particular study both potential profiles still gave rather similar current-voltage characteristics. In the case considered here, the consequences of the tilted potential for the charge transport are significant. This can be seen already for the noninteracting case plotted in Fig. 12(a), where we compare $I-V$ characteristics of both potential profiles at high and zero temperature. The current for the tilted case is significantly smaller than for the flat potential. This is also signaled by a significant decrease of the transmission function (Fig. 12(c)) already for small voltages. 

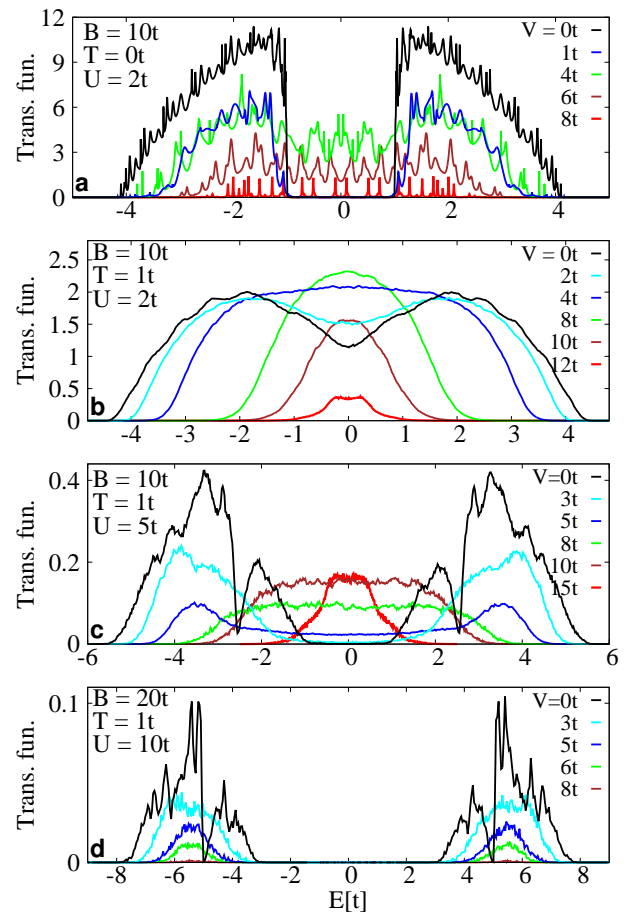

Figure 13. (a-d) Transmission functions for the tilted system potential and different values of $U, T$, and $V$. Other parameters are: lattice size $L=20 \times 20$, coupling $\gamma=0.2 B$, and band half width: (a-c) $B=10 t$ and d) $B=20 t$.

Although a clear difference between tilted and flat geometry is evident for the interacting system as well (see Fig. 12(b)), it is much less dramatic than for $U=0$. This may partially explain the similarities in the currents in Okamoto's work ${ }^{88}$, where $U$ was rather large.

The significant difference of the transport results for $\varepsilon^{\text {flat }}$ and $\varepsilon^{\text {tilt }}$ can be understood by comparing the transmission functions. Whereas the transmission functions for the flat potential in Fig. [6 do not change significantly with voltage unless $V>B$, the tilt of the potential has a profound effect already for small voltages (see Fig. 13). This is further complicated by the strong spatial dependence of the LDOS on $V$ and $U$ that influences the overall transmission differently in central and segregated regions. The low transmission at energies $|E|<U / 2$ for the central region is responsible for the gap in the transmission function but it has to compete with growing segregation that is closing the gap.

The transmission function changes rapidly with increasing voltage for $U=2 t$ at both zero and high temperatures (Fig. 13(a,b)). First, the pseudo-CDW gap closes. This again resembles the effect of lowering $U$. Besides filling the gap, the increasing voltage leads to narrowing of the transmission function. The combination of these two effects at high temperature leads initially to a significant increase of the transmission function around $E=0$. Further increase of the voltage introduces the segregation in the $f$-particle subsystem and the transmission
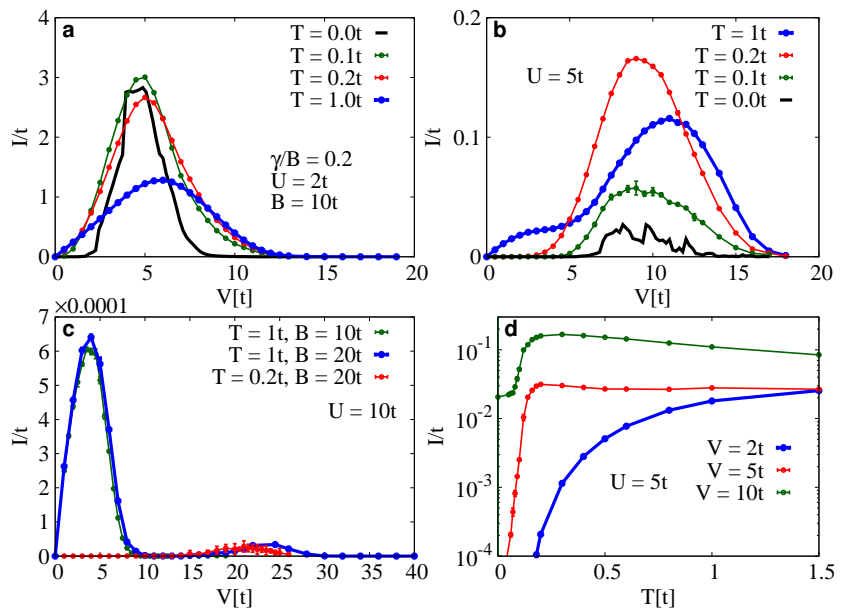

Figure 14. The current-voltage relation for tilted system potential, system size $L=20 \times 20$, coupling $\gamma / B=0.2$ and parameters (a) $U=2 t, B=10 t$; (b) $U=5 t, B=10 t$; and (c) $U=10 t, B=10 t$ and $20 t$ and different temperatures. (d) The transmission function at $E=0$ as a function of temperature for $V=5 t$ and various values of $U$.

function drops down. Consequently, when we compare the $I-V$ characteristics of the flat and tilted potential, plotted in Fig. 12(b), the curves coincide only for $V<U$. The tilt shifts the maximum of the current to lower voltage $(V \sim 5.5 t)$ and a rather steep decline follows for the voltages that are well below $V \sim B$. Therefore, and in contrast to the flat potential, the drop of the current is a consequence of the vanishing transmission function and not of closing the band window $W$.

The situation is more complicated for $U=5 t$. On one hand, the increasing voltage is closing the gap, but, on the other, it pushes the in-gap maxima and Hubbard-like satellites together. This makes the pseudo gap shallower but also effectively broader. As for the weak interaction, with increasing $V$ the transmission function initially increases in the central region (Fig. 13(c)). The transmission function drops again at higher voltages where the segregation starts to form. The voltage dependence of the transmission function for $U=10 t$ is similar to $U=5 t$ (Fig. 13(d)) but the central region is suppressed which is related to the gap in the LDOS of the central layers even for high voltages as seen in Fig. 11(g).

The complicated dependences of the transmission function on the voltage for $U=5 t$ and $U=10 t$ is imprinted in the $I-V$ characteristics plotted in Fig. 14 (b,c). The current for $U=5 t$ and $T=1 t$ has almost a plateau for $1 \lesssim V<5$. This is because two opposite tendencies are active in this region. The increasing width of the Fermi window should increase the current, but it has to compete with the effective increase of the width of the pseudo gap and the decreasing amplitude of the Hubbard-like satellites. The following maximum reflects the increase of the transmission function in the central region (Fig. 13)(c)). This effect is even more pronounced for strong interac- 

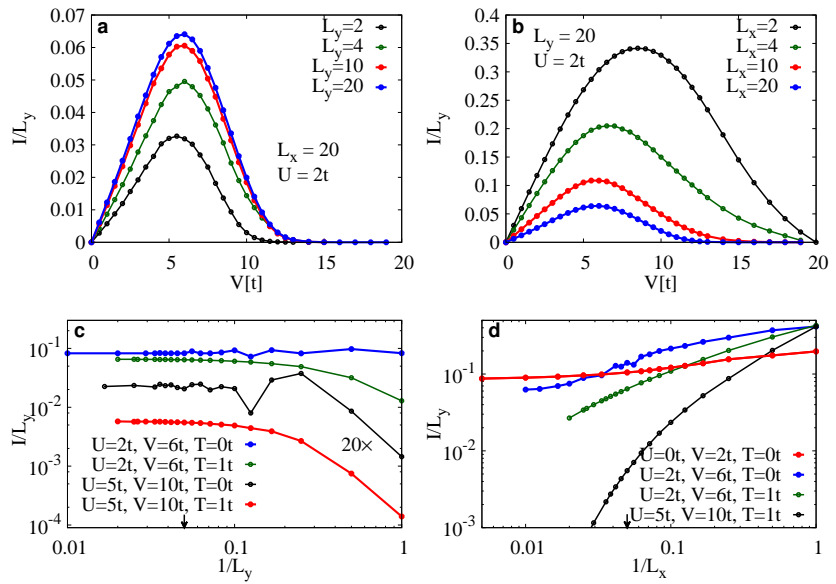

Figure 15. Current densities plotted: (a) as a function of voltage for a system with $L_{x}=20$ and different vertical sizes $L_{y}$ at $T=1 t$; (b) as a function of voltage for a system with $L_{y}=20$ and different numbers of layers $L_{x}$ at $T=1 t$; (c) as a function of inverse linear size $1 / L_{y}$ for system with $L_{x}=20$ and optimal $V$, where the data for $U=5 t$ and $T=1 t$ were multiplied by 20 for the sake of clarity; and (d) as a function of inverse linear size $1 / L_{x}$ for a system with $L_{y}=20$.

tion, resulting in two tiny (see the scale) maxima in the current.

The kinks and sharp steps visible in the $I-V$ characteristics at zero temperature in Fig. 14(a,b) reflect the changes in the ground-state $f$-particle distribution. The MixP does not change from CheP to SegP in a continuous way but rather steplike. We have found that with increasing $V$ the segregated phase always grows by filling and emptying a whole layer. Every such change can lead to a sharp feature in the $I-V$ characteristics.

The presence of the segregated regions significantly influences the temperature dependence of the current as illustrated for $U=5 t$ in Fig. 14(d). We have not observed any sharp increase of the current below critical temperature typical for high-voltage dependencies for the flat potential. This is a consequence of the significant suppression of the Hubbard-like satellites for increasing voltage. A sharp exponential decrease of the current below the critical temperature can be observed for $V=5 t$. With further lowering of the voltage, the sharp steplike decrease changes into a smooth dependence typical for the DPs-OP transition.

\section{System size effects}

The finite-size dependence for the system with tilted potential is quite complicated. This is illustrated in Fig. 15. As for the flat potential case, the current density converges rapidly with increasing $L_{y}$ and is practically saturated for $L_{y}=20$. The saturation is monotonous for high temperature, but it oscillates at $T=0 t$. Small os- cillations are evident even above $L_{y}=20$. The potential tilt has a bigger influence on the scaling in the $x$ direction. The red filled circles in Fig. 15)(d) show that this is true already for the noninteracting case, where the $f$ particles do not play a role. The current density seems to be converging to finite values for weakly and noninteracting cases at zero temperature. However, this convergence is slow and much larger lattices than for the flat potential are needed to confirm the plateau. For the same reason, it is not obvious from the numerical results that the weakly interacting case at high-temperature approaches zero for $L_{x} \rightarrow \infty$. All this shows, that the profile of the system potential has a dramatic effect on the steady-state transport.

\section{CONCLUSIONS}

We have studied charge transport in a model heterostructure, where the central system is described by a Falicov-Kimball model, which is coupled to metallic leads. The method used is based on a combination of a sign-problem-free MC approach and nonequilibrium Green's-functions techniques. Due to its efficiency, this methodology allows us to study large enough structures to approach the condensed phase limit.

We have specifically considered charge transport through a 2D FKM for two types of electrostatic potentials in the system, including a flat and a tilted form. In both cases, the transport characteristic is closely related to the phases of the FKM. For a flat potential, the current signals a DP-OP transition by a sharp steplike increase or decrease of the current depending on the bias voltage. The transition from DPw to DPs can be clearly identified from the properties of the transmission function and it is not affected even by a high voltage. Different regimes of localization can be seen even for high voltages. The tilted potential, on the other hand, introduces new phases including charge segregation. A significant tilt can close the Mott gap and fill in the CDW pseudo gap and therefore significantly affects the current-voltage characteristics.

We have, furthermore, analyzed the dependence of the transport properties on the size of the system and shown that a size of $L_{x}=20 \times 20$ is sufficient for addressing the condensed phase limit. Moreover, we have found that the finite-size scaling of the current is qualitatively different for weak and strong interaction.

In the present paper, we have adopted several simplifications, the lifting of which could be interesting for future investigations. First, the leads were modeled by mutually disconnected semi-infinite chains. Second, it would be interesting to include part of the leads at the interfaces into the system, because the proximity to the system can change the electron distribution in these areas of the leads 62,105 . Finally, we have assumed that in equilibrium the system and the leads have the same effective chemical potential and, therefore, there is no evident effect of electronic charge reconstruction at the 
interfaces ${ }^{106}$. This will typically not be the case in real materials. The extension of the paper to include these aspects will the subject of future work.

\section{ACKNOWLEDGMENTS}

The authors acknowledge support by the state of Baden-Württemberg through bwHPC (High Performance Computing in Baden-Württemberg) and the German Research Foundation (Deutsche Forschungsgemeinschaft) through grant no INST 40/467-1 FUGG. We thank Rainer Härtle for inspiring and very helpful discussions.
1 N. S. Wingreen, A.-P. Jauho, and Y. Meir, Phys. Rev. B 48, 8487 (1993).

2 W. G. van der Wiel, S. De Franceschi, J. M. Elzerman, T. Fujisawa, S. Tarucha, and L. P. Kouwenhoven, Rev. Mod. Phys. 75, 1 (2002)

3 L. DiCarlo, C. M. Marcus, and J. S. Harris, Phys. Rev. Lett. 91, 246804 (2003).

4 S. K. Watson, R. M. Potok, C. M. Marcus, and V. Umansky, Phys. Rev. Lett. 91, 258301 (2003).

5 S. Kohler, J. Lehmann, and P. Hänggi, Phys. Rep. 406, 379 (2005).

6 P. J. Leek, M. R. Buitelaar, V. I. Talyanskii, C. G. Smith, D. Anderson, G. A. C. Jones, J. Wei, and D. H. Cobden, Phys. Rev. Lett. 95, 256802 (2005)

7 F. Hohls, A. C. Welker, C. Leicht, L. Fricke, B. Kaestner, P. Mirovsky, A. Müller, K. Pierz, U. Siegner, and H. W. Schumacher, Phys. Rev. Lett. 109, 056802 (2012).

8 M.-C. Harabula, T. Hasler, G. Fülöp, M. Jung, V. Ranjan, and C. Schönenberger, Phys. Rev. Applied 8, 054006 (2017).

9 M. Galperin, M. A. Ratner, A. Nitzan, and A. Troisi, Science 319, 1056 (2008).

10 N. A. Zimbovskaya and M. R. Pederson, Phys. Rep. 509, 1 (2011).

11 S. Ballmann, R. Härtle, P. B. Coto, M. Elbing, M. Mayor, M. R. Bryce, M. Thoss, and H. B. Weber, Phys. Rev. Lett. 109, 056801 (2012)

12 F. Evers and L. Venkataraman, J. Chem. Phys. 146, 092101 (2017)

13 S. Bouvron, R. Maurand, A. Graf, P. Erler, L. Gragnaniello, M. Skripnik, D. Wiedmann, C. Engesser, C. Nef, W. Fu, C. Schonenberger, F. Pauly, and M. Fonin, NANOSCALE 10, 1487 (2018).

14 E. Burzurí, Y. Yamamoto, M. Warnock, X. Zhong, K. Park, A. Cornia, and H. S. J. van der Zant, Nano Lett. 14, 3191 (2014), pMID: 24801879.

15 S. Gariglio, C. H. Ahn, D. Matthey, and J.-M. Triscone, Phys. Rev. Lett. 88, 067002 (2002)

16 A. Ohtomo, D. Muller, J. Grazul, and H. Hwang, Nature 419, 378 (2002).

17 I. Bozovic, G. Logvenov, M. A. J. Verhoeven, P. Caputo, E. Goldobin, and M. R. Beasley, Phys. Rev. Lett. 93, 157002 (2004).

18 F. Baiutti, G. Gregori, Y. E. Suyolcu, Y. Wang, G. Cristiani, W. Sigle, P. A. van Aken, G. Logvenov, and J. Maier, NANOSCALE 10, 8712 (2018).

19 M. Greiner, O. Mandel, T. Esslinger, T. Hansch, and I. Bloch, Nature 415, 39 (2002).

20 M. Greiner, O. Mandel, T. Hansch, and I. Bloch,
Nature 419, 51 (2002)

21 L. E. Sadler, J. M. Higbie, S. R. Leslie, M. Vengalattore, and D. M. Stamper-Kurn, Nature 443, 312 (2006).

22 G. Clos, D. Porras, U. Warring, and T. Schaetz, Phys. Rev. Lett. 117, 170401 (2016)

23 A. Cavalleri, C. Tóth, C. W. Siders, J. A. Squier, F. Ráksi, P. Forget, and J. C. Kieffer, Phys. Rev. Lett. 87, 237401 (2001)

24 C. Kübler, H. Ehrke, R. Huber, R. Lopez, A. Halabica, R. F. Haglund, and A. Leitenstorfer, Phys. Rev. Lett. 99, 116401 (2007)

25 M. Liu, H. Y. Hwang, H. Tao, A. C. Strikwerda, K. Fan, G. R. Keiser, A. J. Sternbach, K. G. West, S. Kittiwatanakul, J. Lu, S. A. Wolf, F. G. Omenetto, X. Zhang, K. A. Nelson, and R. D. Averitt, Nature 487, 345 (2012)

${ }^{26}$ F. Clerc, C. Battaglia, H. Cercellier, C. Monney, H. Berger, L. Despont, M. G. Garnier, and P. Aebi, J. Phys. Condens. Matter 19, 355002 (2007).

27 L. Perfetti, P. A. Loukakos, M. Lisowski, U. Bovensiepen, M. Wolf, H. Berger, S. Biermann, and A. Georges, New. J. Phys. 10, 053019 (2008).

28 F. Schmitt, P. S. Kirchmann, U. Bovensiepen, R. G. Moore, J.-H. Chu, D. H. Lu, L. Rettig, M. Wolf, I. R. Fisher, and Z.-X. Shen, New. J. Phys. 13, 063022 (2011).

29 M. Rigol, V. Dunjko, V. Yurovsky, and M. Olshanii, Phys. Rev. Lett. 98, 050405 (2007)

30 M. Eckstein and M. Kollar, Phys. Rev. Lett. 100, 120404 (2008)

31 M. Eckstein, M. Kollar, and P. Werner, Phys. Rev. Lett. 103, 056403 (2009)

32 L. Stojchevska, I. Vaskivskyi, T. Mertelj, P. Kusar, D. Svetin, S. Brazovskii, and D. Mihailovic, Science 344, 177 (2014).

33 I. Vaskivskyi, J. Gospodaric, S. Brazovskii, D. Svetin, P. Sutar, E. Goreshnik, I. A. Mihailovic, T. Mertelj, and D. Mihailovic, Sci. Adv. 1, e1500168 (2015).

34 I. Vaskivskyi, I. A. Mihailovic, S. Brazovskii, J. Gospodaric, T. Mertelj, D. Svetin, P. Sutar, and D. Mihailovic, Nat. Commun. 7, 11442 (2016).

35 C. Reichhardt, D. Ray, and C. J. O. Reichhardt, Phys. Rev. B 98, 134418 (2018).

36 H. Breuer and F. Petruccione, The Theory of Open Quantum Systems (Oxford University Press, 2010).

37 H.-P. Breuer, E.-M. Laine, J. Piilo, and B. Vacchini, Rev. Mod. Phys. 88, 021002 (2016).

38 H. Haug and A.-P. Jauho, Quantum Kinetics in Transport and Optics of Semiconductors (Springer, 2008).

39 J. Rammer and H. Rev. Mod. Phys. 58, 323 (1986) 
40 A.

Kamenev

and

A.

Levchenko,

Advances in Physics 58, 197 (2009)

https://doi.org/10.1080/00018730902850504

41 V. Spicka, B. Velicky, and A. Kalvova, Int. J. Mod. Phys. B 28, 1430013 (2014).

42 L. Kadanoff and G. Baym, Quantum Statistical Mechanics (Benjamin, New York, 1962).

43 P. Lipavský, V. Špička, and B. Velický, Phys. Rev. B 34, 6933 (1986).

44 S. Hermanns, K. Balzer, and M. Bonitz, Phys. Scr. 2012, 014036 (2012)

45 J. K. Freericks, V. M. Turkowski, and V. Zlatić, Phys. Rev. Lett. 97, 266408 (2006).

46 H. Aoki, N. Tsuji, M. Eckstein, M. Kollar, T. Oka, and P. Werner, Rev. Mod. Phys. 86, 779 (2014)

47 Y. Tanimura and R. Kubo, J. Phys. Soc. Jpn. 58, 101 (1989)

48 H. Wang and M. Thoss, J. Chem. Phys, 119, 1289 (2003)

49 F. B. Anders and Phys. Rev. Lett. 95, 196801 (2005)

A. Schiller,

50 U. Schollwoeck and S. White, AIP Conf. Proc. 816, 155 (2006).

51 L. Mühlbacher and E. Rabani, Phys. Rev. Lett. 100, 176403 (2008)

52 E. Gull, A. J. Millis, A. I. Lichtenstein, A. N. Rubtsov, M. Troyer, and P. Werner, Rev. Mod. Phys. 83, 349 (2011)

53 R. Härtle, G. Cohen, D. R. Reichman, and A. J. Millis, Phys. Rev. B 88, 235426 (2013)

54 H. T. M. Nghiem and T. A. Costi, Phys. Rev. B 89, 075118 (2014)

55 J. Hubbard, Proc. Royal Soc. Lond. A 276, 238 (1963)

56 L. M. Falicov and J. C. Kimball, Phys. Rev. Lett. 22, 997 (1969)

57 C. Gruber and N. Macris, Helv. Phys. Acta 69, 850 (1996).

58 J. K. Freericks, Phys. Rev. B 70, 195342 (2004)

59 P. Farkasovsky, Acta Phys. Slovaca 60, 497 (2010)

${ }^{60} \mathrm{H}$. Cencarikova and P. Farkasovsky, Condens. Matter Phys. 14, 42701 (2011).

61 J. Freericks, Transport in Multilayered Nanostructures: The Dynamical Mean-Field Theory Approach (Imperial College Press, London, 2006) pp. 1-328.

62 J. K. Freericks, B. K. Nikolić, and P. Miller, Phys. Rev. B 64, 054511 (2001)

63 S. T. F. Hale and J. K. Freericks, Phys. Rev. B 85, 205444 (2012)

64 T. Kaneko, S. Ejima, H. Fehske, and Y. Ohta, Phys. Rev. B 88, 035312 (2013)

65 V. Turkowski and J. K. Freericks, Phys. Rev. B 75, 125110 (2007)

66 A. J. Herrmann, N. Tsuji, M. Eckstein, and P. Werner, Phys. Rev. B 94, 245114 (2016)

67 P. Haldar, M. S. Laad, and S. R. Hassan, Phys. Rev. B 96, 125137 (2017)

68 T. Qin and W. Hofstetter, Phys. Rev. B 97, 125115 (2018)

69 P. Haldar, M. S. Laad, and S. R. Hassan, Phys. Rev. B 94, 081115 (2016)

70 A. Smith, J. Knolle, D. L. Kovrizhin, and R. Moessner, Phys. Rev. Lett. 118, 266601 (2017)

71 A. J. Herrmann, A. E. Antipov, Phys. Rev. B 97, 165107 (2018)
72 J. K. Freericks and V. Zlatic, Rev. Mod. Phys. 75, 1333 (2003)

73 M. M. Maska and K. Czajka, Phys. Rev. B 74, 035109 (2006)

74 M. Žonda, P. Farkašovský, and H. Čenčariková, Solid State Commun. 149, 1997 (2009).

75 M. Žonda, Phase Transit. 85, 96 (2012)

76 L. Huang and L. Wang, Phys. Rev. B 95, 035105 (2017).

77 V. Turkowski and J. K. Freericks, Phys. Rev. B 71, 085104 (2005)

78 E. Arrigoni, M. Knap, and W. von der Linden, Phys. Rev. Lett. 110, 086403 (2013)

79 A. E. Antipov, Y. Javanmard, P. Ribeiro, and S. Kirchner, Phys. Rev. Lett. 117, 146601 (2016)

80 M. Hohenadler and F. F. Assaad, Phys. Rev. Lett. 121, 086601 (2018)

81 M. M. Maśka, R. Lemański, J. K. Freericks, and C. J. Williams, Phys. Rev. Lett. 101, 060404 (2008)

82 J. K. Freericks, V. M. Turkowski, and V. Zlatić, Phys. Rev. B 71, 115111 (2005)

83 A.-P. Jauho, N. S. Wingreen, and Y. Meir, Phys. Rev. B 50, 5528 (1994)

84 D. Ryndyk, R. Gutiérrez, B. Song, and G. Cuniberti, Energy Transfer Dynamics in Biomaterial Systems. Springer Series in Chemical Physics, Vol. 93 (2009).

85 M. Č́žek, M. Thoss, and W. Domcke, Phys. Rev. B 70, 125406 (2004).

86 Y. Motome and N. Furukawa, J. Phys. Soc. Jpn. 68, 3853 (1999)

87 V. Cerný, J. Optimiz. Theory App. 45, 41 (1985).

88 S. Okamoto, Phys. Rev. Lett. 101, 116807 (2008).

89 G. Watson and R. Lemanski, J. Phys.-Condens. Mat. 7, 9521 (1995)

90 R. Lemański, J. K. Freericks, and G. Banach, Phys. Rev. Lett. 89, 196403 (2002)

91 U. Brandt and C. Mielsch, Z. Phys. B Con. Mat. 75, 365 (1989).

92 U. Brandt and C. Mielsch, Z. Phys. B Con. Mat. 79, 295 (1990)

93 U. Brandt and C. Mielsch, Z. Phys. B Con. Mat. 82, 37 (1991).

94 L. Chen, J. K. Freericks, and B. A. Jones, Phys. Rev. B 68, 153102 (2003)

95 S. R. Hassan and H. R. Krishnamurthy, Phys. Rev. B 76, 205109 (2007).

96 O. P. Matveev, A. M. Shvaika, and J. K. Freericks, Phys. Rev. B 77, 035102 (2008)

97 R. Lemański and K. Ziegler, Phys. Rev. B 89, 075104 (2014)

98 P. A. Lee and

Phys. Rev. Lett. 47, 882 (1981)

99 O. P. Matveev, A. M. Shvaika, T. P. Devereaux, and J. K. Freericks, Phys. Rev. B 94, 115167 (2016)

100 N. C. Murphy, R. Wortis, and W. A. Atkinson, Phys. Rev. B 83, 184206 (2011).

101 J. Perera and R. Wortis, Phys. Rev. B 92, 085110 (2015)

02 F. Evers and A. D. Mirlin, Rev. Mod. Phys. 80, 1355 (2008)

103 J. K. Freericks, C. Gruber, and N. Macris, Phys. Rev. B 60, 1617 (1999).

104 M.-T. Tran, Phys. Rev. B 73, 205110 (2006)

105 S. Okamoto and A. J. Millis, Phys. Rev. B 70, 241104 (2004)

106 S. Okamoto and A. Millis, Nature 428, 630 (2004) 